\title{
Large moments in external fields of cubic group symmetry
}

\author{
V. A. Kalatsky ${ }^{a}$ and V. L. Pokrovsky ${ }^{a, b}$ \\ ${ }^{a}$ Department of Physics, Texas A\& M University, College Station, Texas 77843-4242 \\ ${ }^{b}$ Landau Institute for Theoretical Physics, Kosygin str.2, Moscow 117940, Russia
}

(May 27, 2017)

We predict that large moments $J$, placed into a crystal field with the cubic point symmetry group, differ by their spectrum and magnetic properties. E. g., properties of the odd-integer moments are different from those of the eveninteger. The effect is due to Berry's phases gained by the moment, when it tunnels between minima of the external field. Two cases of the group $\mathbf{O}$ are classified, namely, 6- and 8fold coordinations. The spectrum and degeneration of energy levels depend on a remainder $\{J / n\}$, where the divisor $n=4$ and 3 for 6 -fold and 8 -fold coordination respectively. Large moments in the cubic environment can be realized by diluted alloys $\mathrm{R}_{1-x} \mathrm{R}_{x}^{\prime} \mathrm{Sb}$, where $\mathrm{R}=\mathrm{Lu}, \mathrm{La}$, and $\mathrm{R}^{\prime}=\mathrm{Tb}, \mathrm{Dy}$, Ho, Er.

PACS numbers: 03.65.Bz, 75.30.Cr, 75.10.Dg

It has been shown on several examples (dependence of the Haldane gap in anti-ferromagnetic spin chain [1] and in 2D isotropic Heisenberg antiferromagnets [2], suppression of tunneling in magnetic particles [3], magnetization of ions in external fields (国) that systems with large integer moments may behave differently from ones with large half-integer moments although the values of the moments are close to each other. Haldane pointed out in [2] that there may be a difference between odd and even spins as well. All of them are due to Berry's connection [5]. In this paper we demonstrate systems where further differentiation on the moment values occurs.

The systems under study are large moments in strongly anisotropic external fields, e.g, crystal electric field with deep wells at certain crystallographic directions. We consider systems of the octahedron symmetry with 6 - and 8 -fold coordination. They show a subtle partition on the moment values.

In classical description, the angular moment is a vector of a fixed length $J$ determined by the direction of a unit vector $\mathbf{n}$. Let us assume that an ion with a large moment is placed into a crystalline electric field (CEF). Due to symmetry the moment may be localized in 6 symmetrical positions (the easy positions of $\mathrm{CEF}$ ). We choose following spherical coordinates $(\theta, \phi)$ for the positions: $1-(0,0), 2-(\pi, 0), 3-(\pi / 2,0), 4-(\pi / 2, \pi), 5-(\pi / 2, \pi / 2)$, and $6-(\pi / 2,3 \pi / 2)$. Such a configuration will be called octahedral or 6 -fold. The ground state of the system is 6 -fold degenerate if tunneling between the potential wells is neglected. If one allows the moment to tunnel to the nearest wells along the geodesics on the unit sphere (see Fig. 1), the degeneracy will be lifted at least partially.

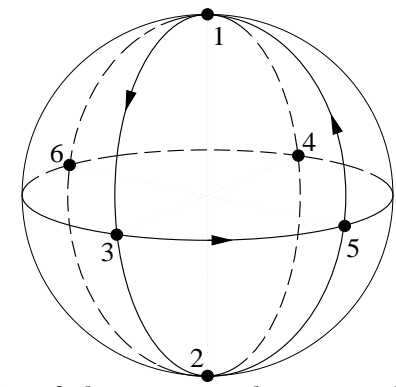

FIG. 1. Paths of the spin on the unit sphere between the easy positions of the field. The case of the 6 -fold coordination.

A formal description of such a system is given by a Hamiltonian $(\mathcal{H})$ with the following matrix elements:

$$
\begin{aligned}
h_{i i} & =0, i=1,2, \ldots 6, \\
h_{i j} & =0,|i-j|=1, i+j=3,7,11, \\
\left|h_{i j}\right| & =|w|, \text { for other } 1 \leq i, j \leq 6
\end{aligned}
$$

where we adopted the enumeration shown on Fig. 1.

The moment's wave-function, upon tunneling from one minimum $(i)$ to another $(j)$, may gain a geometric phase shift $\phi\left(C_{i j}\right)$ which depends on the tunneling path $C_{i j}$ on the unit sphere (the $\mathbf{n}$-space): $\phi\left(C_{i j}\right)=\int_{C_{i j}} \mathbf{A}(\mathbf{n}) \mathrm{d} \mathbf{n}$. The vector field $\mathbf{A}$ is called Berry's connection. Specially for the problem of semi-classical moments it was calculated in 5., 1 . The appropriate geometric phase was first discovered by Berry [5].

The phase shifts $\phi\left(C_{i j}\right)$ as well as the connection $\mathbf{A}$ are not gauge invariant: $\mathbf{A}(\mathbf{n}) \rightarrow \mathbf{A}(\mathbf{n})-\nabla f(\mathbf{n})$, where $f(\mathbf{n})$ is an arbitrary continuous function, the spins' eigenfunctions transform as: $\psi(\mathbf{n}) \rightarrow e^{i f(\mathbf{n})} \psi(\mathbf{n})$. In our case (well localized states) it reduces to: $\psi_{k} \rightarrow e^{i f_{k}} \psi_{k}$, where $f_{k}=f\left(\mathbf{n}_{k}\right), \mathbf{n}_{k}(k=1, \ldots, 6)$ are the positions of the potential wells. However, the phase $\gamma(C)$ accumulating at circulation along a closed circuit $C$ is invariant:

$$
\gamma(C)=\oint_{C} \mathbf{A}(\mathbf{n}) \cdot \mathrm{d} \mathbf{n}= \pm J \Omega(C),
$$

where $\Omega(C)$ is the solid angle subtended by a closed path $C$ on the unit sphere at its origin. The phase shifts $\phi\left(C_{i j}\right)$ change to $\phi\left(C_{i j}\right)+f_{j}-f_{i}$ by the gauge transformation. Therefore, $\phi\left(C_{i j}\right)$ can be any set of numbers satisfying the invariant relationships (2). The Hamiltonian also is not invariant, it transforms as: $\mathcal{H} \rightarrow \mathcal{U} \mathcal{H} \mathcal{U}^{\dagger}$, where $\mathcal{U}$ is a diagonal matrix with matrix elements $u_{k k}=\exp \left(i f_{k}\right)$.

Tunneling trajectories divide the sphere into 8 plaquettes. Each of them represents a minimal non-trivial 
closed loop and subtends the solid angle of $\pi / 2$ (e. $g$. closed path $1-3-5-1$ on Fig. 11). The 8 plaquettes give 8 equations for the phase shifts:

$$
\phi_{i j}+\phi_{j k}+\phi_{k i}=J\left(\frac{\pi}{2} \bmod 4 \pi\right),
$$

where $\phi_{i j}$ is the phase shift upon tunneling from easy position $i$ to easy position $j$, and set $\{(i, j, k)\}$ enumerates the plaquettes. Only 7 out of the 8 equations are independent which leads to 5 degrees of freedom over 12 phases. This number (five) is equal to the number of independent parameters in a general gauge transformation ( $\operatorname{six}$ ) minus one corresponding to a common phase factor. Note that the Hamiltonian (1) is not invariant under the action of the octahedron group realized as a subgroup of the permutation group on the 6 states. What leaves the Hamiltonian invariant is a rotation $(\mathcal{R})$ accompanied by an appropriate gauge transformation $(\mathcal{U})$ : $\mathcal{H}=\mathcal{U} \mathcal{R} \mathcal{H} \mathcal{R}^{T} \mathcal{U}^{\dagger}$, where $\mathcal{U}$ depends on $\mathcal{R}$, and $J$.

Hamiltonian (11) can be diagonalized leading to the following 6 eigenvalues:

$$
\begin{aligned}
E_{k}(J) & =(-1)^{k} 2 w \chi(\pi(J+2 k)), k=0, \ldots, 5, \\
\chi(x) & =\cos \frac{2 x}{3} \cos \frac{x}{2}-\left(\cos ^{2} \frac{x}{3}+\sin ^{2} \frac{2 x}{3} \sin ^{2} \frac{x}{2}\right)^{1 / 2} .
\end{aligned}
$$

The ground state energies and their degeneracies fall into 5 classes which are gathered in Table 1 . The classes differ by their ground-state energies, degeneracies of the ground-state, and excitation energies. All these will lead to experimentally observable dissimilarities between systems falling into the different classes for $k_{\mathrm{B}} T<w, e . g$, thermodynamical properties or response to applied magnetic field.

The number of the classes and the moments' values of each class can be easily found without detailed analysis of the Hamiltonian for the external fields of the perfect polyhedra group symmetries (perfect Hamiltonians). Let us assume that the parameter space (unit sphere) can be covered with $p$ plaquettes (the number of the faces of a perfect polyhedron). Then, each plaquet subtends a solid angle of $4 \pi / p$ at the origin of the sphere and Berry's phase for each loop is $4 \pi J / p$. This gives a pe$\operatorname{riod}$ of $J: J_{p}=p / 2$. The spectra of the systems differing by transformation $\gamma(C) \rightarrow-\gamma(C)$ must be identical due to time-reversal symmetry. It leads to equivalence of $J=J_{p} n+m / 2$ and $J=\left|J_{p} n-m / 2\right|, n, m=0,1,2, \ldots$ Hence, we can classify equivalent moments in the following manner:

$$
J_{m}=\left|J_{p} n \pm \frac{m}{2}\right|, n=0,1,2, \ldots ; m=0,1, \ldots, J_{p},
$$

and the number of the classes is $C_{p} \equiv J_{p}+1=p / 2+1$. The classes can be conveniently labeled by the integer $m+1$. E. g., for the 6 -fold coordination (octahedron) one has: $p=8, C_{8}=5$. Another example is a planar external field (degenerate polyhedron with two faces polygon): $p=2, C_{2}=2$, and $J_{m}=|n \pm m / 2|, m=0,1$. This case was studied in [4] for polygon=square and in [3] for polygon=line (easy plane, easy axis anisotropy).

There is an extra symmetry in systems with a perfect Hamiltonian which may relate energy levels of different classes or inside of a class. It is the change of sign of $w$. This transformation should inverse energy levels inside of each class. On the other hand, all physical quantities depend only upon gauge invariant combinations of a type $w^{k} \cos \left(J \Omega_{k}\right)$, where $\Omega_{k}$ is the solid angle subtended by a closed loop of $k>1$ walks along the geodesics. In some cases (polyhedra made of even-sided faces: cube, square) $k$ is always even and this leads to the symmetry of the levels inside of each class, namely, the levels must come in pairs of opposite sign $\pm E$. In other occurrences (polyhedra made of odd-sided faces: octahedron, tetrahedron) $k$ may be both odd and even. Then simultaneous change of sign of $w \rightarrow-w$ and shift of $J \rightarrow J+p / 4$ leaves the invariant combinations unchanged. Therefore, each level $E$ in the class of $J$ has its counterpart $-E$ in the class of $J+p / 4$. If $J$ and $J+p / 4$ are equivalent, their spectrum is symmetric (e.g., class 3 in Table I).

Let us consider the magnetic response of different spins. We assume that magnetic field $\mathbf{H}$ is small, i.e., $g J \mu_{B}|\mathbf{H}| \ll U(J)$ where $U(J)$ is the variation of the CEF potential. The magnetic part of the Hamiltonian is a diagonal matrix:

$\mathcal{H}_{\mathrm{M}}=J \operatorname{diag}\left(-h_{z}, h_{z},-h_{x}, h_{x},-h_{y}, h_{y}\right), h_{\alpha}=g \mu_{B} H_{\alpha}$,

where $g$ is the gyro-magnetic ratio and $\alpha=x, y, z$. The full Hamiltonian $\left(\mathcal{H}+\mathcal{H}_{M}\right)$ can be diagonalized for some symmetric directions of the field, e. $g$., along an easy direction $(0,0,1)$. We present the ground state energies up to the second order in magnetic field for all classes in Table [II.

Note that class 2 has anisotropic magnetic moment, and classes 1, 2, and 3 have anisotropic magnetic susceptibility in the ground state. Some classes $(2,3$, and 4) have non-zero magnetization in the ground-state. The low temperature behavior of these classes should be strikingly different from those without magnetization. We will discuss it later.

For the 8-fold or cubic case the easy positions of CEF, on the unit sphere, can be chosen in the following way: $( \pm \arccos (1 / \sqrt{3}),(2 k+1) \pi / 4), k=0,1,2,3$ and enumerated as $(4 k+3 \pm 1) / 2$ (see Fig. 2). The parameter space can be covered with $p=6$ plaquettes and we should expect $C_{6}=4$ classes with members $J_{m}=|3 n \pm m / 2|$, $m=0,1,2,3$ all sets of levels are symmetric. The solid angle subtended by each plaquet is $2 \pi / 3$ which gives 6 ( 5 independent) equations for the phase shifts. 


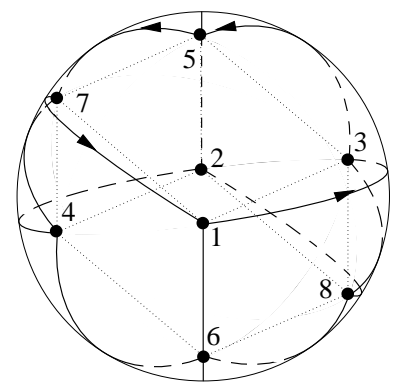

FIG. 2. Paths of the spin on the unit sphere between the easy positions of the field. The case of the 8-fold coordination.

The eigenvalues of the Hamiltonian can be expressed in the following closed form:

$$
\begin{aligned}
E_{k}^{ \pm} & = \pm 2 w \xi(\pi(J+3 k)), k=0,1,2,3, \\
\xi(x) & =\left(3+2 \cos x \cos \frac{2 x}{3}+4 \cos \frac{x}{2} \cos \frac{x}{3} f(x)\right)^{\frac{1}{2}} \\
f(x) & =\left(4 \sin ^{2} \frac{x}{2} \sin ^{2} \frac{x}{3}+1\right)^{\frac{1}{2}} .
\end{aligned}
$$

The ground state energies of the cubic coordination classes and their degeneracies are presented in Table III. The responses of different classes to applied magnetic field should be quite different. To demonstrate this we apply magnetic field along one of the easy direction of the $\mathrm{CEF}, e$. g., the first position on Fig. 2 $(\mathbf{H}=H(1,1,1) / \sqrt{3})$. The Zeeman term of the Hamiltonian, in the chosen parameterization, is $-h J(3,-3,1,-1,-1,1,1,-1) / 3$. Taylor expansions of the ground state energies up to the second order in magnetic field are given in Table IV. There are three classes with non-zero magnetization in the ground state. Class 4 has anisotropic magnetic moment, and class 3 has anisotropic magnetic susceptibility in the ground state.

There exists one more type of the coordination in the cubic CEF - 12-fold. The 12-fold coordination is realized if the minima of the CEF are located at the centers of the cube's edges and can be parameterized on the unit sphere as $(k \pi / 4,(2 k+l-1) \pi / 4), k=1,2,3$, $l=0,1,2,3$. Unfortunately, the tunneling trajectories do not coincide with the geodesics. As a consequence, the number of classes, in general, is infinite. We will not consider the 12 -fold coordination here due to its strong dependence on the CEF parameters.

Now we proceed to the temperature variation of the magnetic response. For temperatures $k_{\mathrm{B}} T>w$ large moments become purely classical and exhibit Curie magnetic susceptibility:

$$
\chi_{\mathrm{C}}=\left(\mu_{\mathrm{B}} g J\right)^{2} /\left(3 k_{\mathrm{B}} T\right) .
$$

For temperatures $k_{\mathrm{B}} T<w$ the quantum effects change the response drastically. The susceptibility saturates for the classes without magnetic moment in the ground state to the value:

$$
\chi_{s}=\frac{1}{g_{0}} \sum_{i=1}^{g_{0}} \chi_{i}
$$

where $g_{0}$ is the degeneracy of the ground state and $\chi_{i}$ is the susceptibility of the $i$ th member of the ground-state multiplet. For the classes with the magnetic moment in the ground state Curie susceptibility persists at $k_{\mathrm{B}} T<$ $w$, but its slope is different:

$$
\chi_{l}=\frac{1}{g_{0}} \sum_{i=1}^{g_{0}} m_{i}^{2} /\left(k_{\mathrm{B}} T\right),
$$

where $m_{i}$ is the moment of the $i$ th member of the groundstate multiplet. We collect the low temperature susceptibilities of the 6- and 8-fold systems in the last column of Tables II and IV respectively (a common factor is $\left.\left(g J \mu_{\mathrm{B}}\right)^{2}\right)$. The exact dependencies of the susceptibility versus inverse temperature are given on Fig. 3 and 1 for the 6 - and 8-fold coordinations respectively. The susceptibility is isotropic in all cases due to the cubic symmetry.

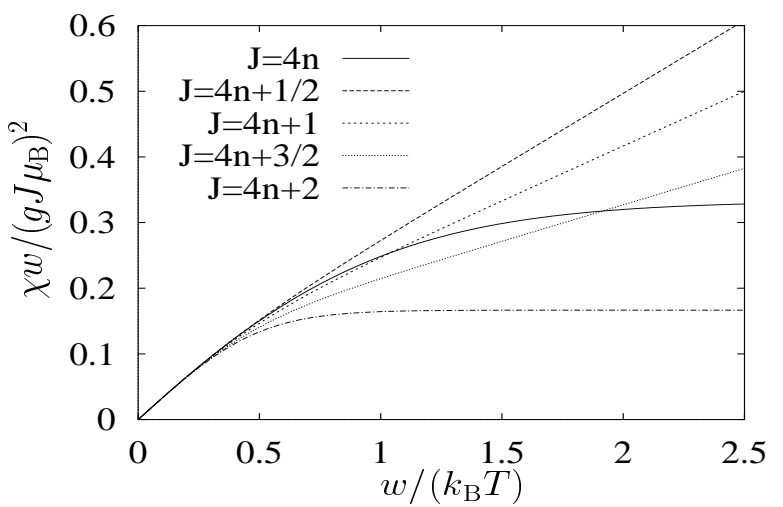

FIG. 3. Susceptibility versus inverse temperature in the 6-fold coordination

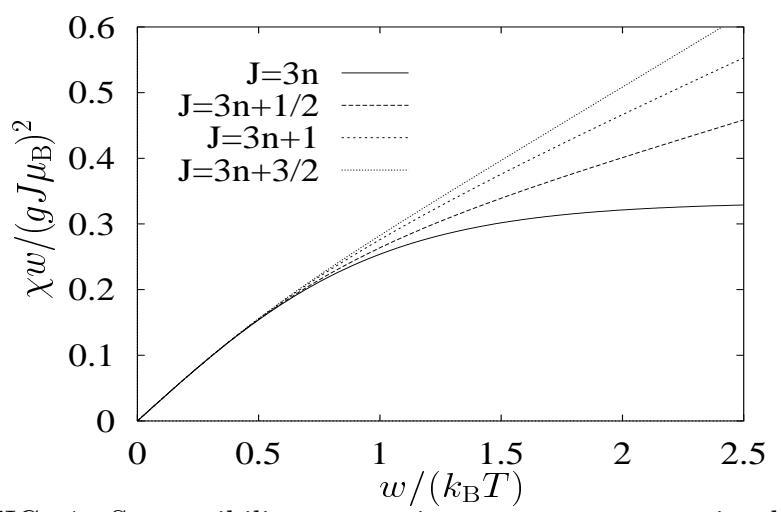

FIG. 4. Susceptibility versus inverse temperature in the 8-fold coordination

Now, let us deliberate experimentally observable differences among the classes. First of all we need to have a rare-earth compound with the octahedral coordination. The general form of the CEF potential of the octahedral coordination, as it was shown in [6], reads:

$$
U\left(J_{x}, J_{y}, J_{z}\right)=A\left(J_{x}^{4}+J_{y}^{4}+J_{z}^{4}-\frac{3}{5} J^{4}\right)+
$$




$$
B\left(J_{x}^{6}+J_{y}^{6}+J_{z}^{6}+30 J_{x}^{2} J_{y}^{2} J_{z}^{2}-\frac{5}{7} J^{6}\right) / J^{2},
$$

where $A$ and $B$ are CEF constants. Higher order invariants are irrelevant for the most interesting situation of the rare-earth $(\mathrm{R})$ ions with $4 f$-electrons.

A general analysis of the CEF potential (9) shows that the plane of parameters $(A-B)$ is divided into three sectors by the regions of stability of the 6-, 8-, and 12 -fold coordinations:

$$
\begin{array}{r}
\text { 6-fold }\left(\Gamma_{c}\right): A<B / 3, A<-3 B / 2, \\
\text { 8-fold }\left(\Gamma_{c}^{v}\right): A>B / 3, A>35 B / 6, \\
\text { 12-fold }\left(\Gamma_{c}^{f}\right): \quad-3 B / 2<A<35 B / 6 .
\end{array}
$$

where we enclose in parentheses the Bravais lattices of the cubic system which realize the respective coordinations. An example of the 6-fold coordination is RSb rock-salt structured family of compounds.

The experimental observation of the discussed effects can be done on dilute alloys of the type $\mathrm{R}_{x} \mathrm{R}_{1-x}^{\prime} \mathrm{Sb}$, where $\mathrm{R}^{\prime}=\mathrm{La}, \mathrm{Lu}$ (non-magnetic), and $\mathrm{R}=\mathrm{Tb}(J=6$, class 5$)$, Dy $(J=15 / 2$, class 2$)$, Ho $(J=8$, class 1$)$ or any other equivalent set of elements given in Table $\square$. Besides magnetization and susceptibility measurements discussed above one can study the EPR in these alloys. The spectral measurements can display the universal ratios of the resonance frequencies and check the values of the magnetic momenta predicted by the theory. Our preliminary estimates show that Jahn-Teller effect is not essential in the conditions of the experiment proposed in this article at least at temperatures higher than $0.1 \mathrm{~K}$. The analysis of Jahn-Teller effect will be done elsewhere.

This work was supported by the NSF grant DMR9705812. We thank P.C. Canfield who indicated us RSb compounds' family.

[1] F. D. M. Haldane, Phys. Rev. Lett. 50, 1153 (1983); Phys. Lett. A 93, 464 (1983).

[2] F. D. M. Haldane, Phys. Rev. Lett. 61, 1029 (1988).

[3] D. Loss, D. P. DiVincenzo, and G. Grinstein, Phys. Rev. Lett. 69, 3232 (1992)

[4] V. A. Kalatsky, E. Müller-Hartmann, V. L. Pokrovsky, and G. S. Uhrig, to be published in PRL.

[5] M. V. Berry, Proc. Roy. Soc. London, Ser. A 392,45 (1984)

[6] M. T. Hutchings, in Solid State Physics, edited by F. Zeitz and D. Turnbull (Academic, New York, 1964), Vol. 16, p. 227.

TABLE I. The ground state energies and their degeneracies of the 6 -fold coordination for $w>0(n=1,2,3, \ldots)$

\begin{tabular}{llll}
\hline \hline Class & Moment $(J)$ & Ground state & First excited
\end{tabular}

\begin{tabular}{lllll} 
& & energy & degeneracy & state energy \\
\hline 1 & $4 n$ & $-2 w$ & 2 & 0 \\
2 & $4 n \pm 1 / 2$ & $-\sqrt{2} w$ & 4 & $2 \sqrt{2} w$ \\
3 & $4 n \pm 1$ & $-2 w$ & 3 & $2 w$ \\
4 & $4 n \pm 3 / 2$ & $-2 \sqrt{2} w$ & 2 & $\sqrt{2} w$ \\
5 & $4 n+2$ & $-4 w$ & 1 & 0 \\
\hline \hline
\end{tabular}

TABLE II. The ground state energies in magnetic field along $(0,0,1)$ direction and the low temperature magnetic susceptibilities of the 6 -fold coordination classes $\left(\beta=1 /\left(k_{\mathrm{B}} T\right)\right)$

\begin{tabular}{lll}
\hline \hline Class & Ground state energy & Susceptibility \\
\hline 1 & $-2 w-J^{2} h^{2} /(3 w)$ & $1 /(3 w)$ \\
2 & $-\sqrt{2} w-2 J h / 3-\sqrt{2} J^{2} h^{2} /(27 w)$ & $2 \beta / 9$ \\
3 & $-2 w-J h / 2-J^{2} h^{2} /(16 w)$ & $\beta / 6$ \\
4 & $-2 \sqrt{2} w-J h / 3-\sqrt{2} J^{2} h^{2} /(27 w)$ & $\beta / 9$ \\
5 & $-4 w-J^{2} h^{2} /(12 w)$ & $1 /(6 w)$ \\
\hline \hline
\end{tabular}

TABLE III. The ground state energies and their degeneracies of the 8-fold coordination for $w>0(n=1,2,3, \ldots)$

\begin{tabular}{lllll}
\hline \hline Class & Moment $(J)$ & \multicolumn{2}{c}{ Ground state } & First excited \\
& & energy & degeneracy & state energy \\
\hline 1 & $3 n$ & $-3 w$ & 1 & $-w$ \\
2 & $3 n \pm 1 / 2$ & $-\sqrt{6} w$ & 2 & 0 \\
3 & $3 n \pm 1$ & $-2 w$ & 3 & 0 \\
4 & $3 n \pm 3 / 2$ & $-\sqrt{3} w$ & 4 & $\sqrt{3} w$ \\
\hline \hline
\end{tabular}

TABLE IV. The ground state energies in magnetic field along $(1,1,1)$ direction and the low temperature magnetic susceptibilities of the 8 -fold coordination classes

\begin{tabular}{lll}
\hline \hline Class & Ground state energy & Susceptibility \\
\hline 1 & $-3 w-J^{2} h^{2} /(6 w)$ & $1 /(3 w)$ \\
2 & $-\sqrt{6} w-J h / 3-\sqrt{6} J^{2} h^{2} /(27 w)$ & $\beta / 9$ \\
3 & $-2 w-J h / 2-13 J^{2} h^{2} /(144 w)$ & $\beta / 6$ \\
4 & $-\sqrt{3} w-2 J h / 3-\sqrt{3} J^{2} h^{2} /(54 w)$ & $2 \beta / 9$ \\
\hline \hline
\end{tabular}

TABLE V. Distribution of $R^{3+}$ magnetic ions among the classes of the 6 - and 8-fold coordinations.

\begin{tabular}{llllll}
\hline \hline Class & 1 & 2 & 3 & 4 & 5 \\
\hline 6-fold & Pm Ho & Ce Nd Sm Gd & & & Pr Eu \\
& & Dy Er Yb & & & Tb Tm \\
\hline 8-fold & Pr Eu & Ce Gd Yb & Pm Ho & Nd Sm \\
& Tb Tm & & & Dy Er & \\
\hline \hline
\end{tabular}

